\title{
THE ABSORPTION AND EXCRETION OF TOCOPHEROL IN LAENNEC'S CIRRHOSIS ${ }^{1}$
}

\author{
BY GERALD KLATSKIN AND DAVID W. MOLANDER ₹
}

\author{
(From the Department of Internal Medicine, Yale University School of Medicine, \\ New Haven, Conn.)
}

(Submitted for publication August 4, 1951; accepted October 29, 1951)

The plasma tocopherol level is often low in cirrhosis $(1,2)$. Whether such low values reflect alterations in the absorption, utilization or excretion of tocopherol related to liver damage, or are the result of a decreased dietary intake is not known. Previous attempts to investigate tocopherol metabolism in liver disease have been limited to tests of tocopherol tolerance based on plasma curves $(1,2)$ and have shed little light on the problem, largely because tolerance tests cannot be interpreted in terms of absorption and utilization without parallel studies of excretion and storage. The present report is concerned with the results of such studies in Laennec's cirrhosis. These have been made possible by the recent development of analytical methods for the determination of tocopherol in excreta.

In a preliminary investigation (1) of a group of subjects with a variety of liver diseases, including Laennec's cirrhosis, it was found that the plasma tocopherol level was significantly lower than in young healthy adults, but that it did not differ significantly from that of randomly-selected, hospitalized convalescent controls without evidence of liver disease. Moreover, there was no correlation between the plasma level and the degree of hepatic dysfunction, suggesting that low levels might be related to extrahepatic factors. Oral tocopherol tolerance tests were carried out to exclude impairment of absorption as one of these factors. These revealed relative flat plasma curves, but the lack of any correlation between the height of the curve and the original plasma level did not appear to be consistent with the interpretation that low plasma concentrations were the result of impaired absorption. Attempts to investigate tocopherol utilization by means of parenteral tolerance tests

\footnotetext{
1 Supported by research grants from Hoffmann-La Roche, Inc., Nutley, N. J., and the National Institutes of Health, U. S. Public Health Service.

2 Present address: Pack Medical Group, New York, N. $\mathbf{Y}$.
}

were unsuccessful, because none of the available preparations of tocopherol could be used intravenously, and none was freely absorbed from muscle. No definite conclusions could be drawn from these observations, but it was suggested that the low plasma levels seen in some subjects with liver disease might be the result of a reduced dietary intake of tocopherol.

The present study was limited to Laennec's cirrhosis, since there was reason to believe that alterations in tocopherol metabolism might differ in the various hepatic disease groups previously investigated. The results indicate that there is no impairment of tocopherol absorption or storage in the cirrhotic, and suggest that low plasma tocopherol levels are probably indicative of depleted tissue stores related to a deficient dietary intake.

\section{MATERIAL AND METHODS}

Subjects. The combined study of tocopherol saturation, tolerance and excretion described below was carried out in 11 subjects with Laennec's cirrhosis, eight normal healthy adults, and seven randomly-selected, hospitalized, convalescent controls. Additional data on plasma tocopherol concentration and on tocopherol tolerance tests were obtained in 22 other cirrhotics, 23 normals, and 53 convalescents.

The diagnosis of Laennec's cirrhosis was clearly evident from the clinical and laboratory findings in every instance, and was confirmed by needle biopsy of the liver in all but one case. On the whole the disease was in an advanced stage when these studies were carried out. The normal healthy adults were medical students, physicians, nurses, and laboratory technicians between the ages of 20 and 40. The hospitalized controls were convalescent from a variety of diseases at the time of the study. Liver disease was excluded in both control groups on the basis of the history and physical findings and the result of multiple liver function tests.

Diets. The cirrhotics were on an experimental diet which provided 1 gram of protein per kilogram, 100 grams of fat, and sufficient carbohydrate to raise the total intake to 30 calories per kilogram of body weight. The total tocopherol content of several such diets was calculated from the tables published by Harris, Quaife and Swanson 
(3) and was found to range between 17.87 and $25.97 \mathrm{mg}$., averaging $21.03 \mathrm{mg}$. per day.

The normal controls, with few exceptions, ate their meals in the staff dining room of the hospital. The tocopherol content of the diet provided there on five randomly-selected days varied between 22.14 and 42.73 mg., averaging $33.53 \mathrm{mg}$. per day.

The convalescent controls were all on the routine hosiptal diet while under investigation. Its tocopherol content was found to range between 25.52 and $43.50 \mathrm{mg}$. on five randomly-selected days, and averaged $30.76 \mathrm{mg}$.

Analytical techniques. Plasma tocopherol concentration was determined on oxalated venous blood by the method of Quaife and Biehler (4). Due to the large volume of plasma required it was not possible to carry out analyses in duplicate. Recoveries of 98.7 to 101.5 per cent were obtained when d-alpha-tocopherol was added to serum in amounts equivalent to 0.5 to $2.0 \mathrm{mg}$. per cent.

Fecal tocopherol was determined by a modification (5) of the method described by Quaife and Harris (6) for the analysis of total tocopherol in foods. In brief the specimens were homogenized, quick-frozen in dry-ice and alcohol, dehydrated under vacuum, and then extracted with hot ethanol in a Soxhlet apparatus. The extracted lipids were then transferred to petroleum ether, evaporated, and then distilled under high vacuum in an analytical molecular still. The distillate was taken up in ethanol, hydrogenated to remove non-tocopherol reducing substances, and then analyzed for total tocopherol photometrically, employing the Emmerie-Engel ferric chloride reductionbipyridine reaction. All determinations were carried out in duplicate samples, which did not vary by more than \pm 3 per cent. Recoveries of 98 to 103 per cent were obtained following molecular distillation of pure d-alphatocopherol in amounts ranging from 10 to 1,000 gamma. On addition of d-alpha-tocopherol to 100 gram aliquots of feces, recoveries varied from 92 to 99 per cent with additions of 25 to $1,000 \mathrm{mg}$., but fell to 73 to 89 per cent when the addition was as small as $10 \mathrm{mg}$.

The method employed in analyzing the plasma and feces for tocopheryl esters was based on a saponification technique carried out in an atmosphere of nitrogen, following preliminary evacuation of air from the samples and reagents (5).

All samples of plasma and feces were stored in a dryice refrigerator at temperatures well below $-20^{\circ} \mathrm{C}$. and were usually analyzed within a few days of their collection. It has been shown that tocopherol is stable under these conditions for at least eight weeks (7).

Tocopherol tolerance tests. Following the administration of $500 \mathrm{mg}$. of dl-alpha-tocopheryl acetate (457 mg. of free tocopherol) in tablet form, ${ }^{3}$ specimens of blood were drawn for tocopherol analysis at $0,3,6,9,12$, and 24 hours. The test was begun in the early morning with the subject in the fasting state, but only the 0 and 24 hour specimens were collected under these conditions, the subject being allowed to eat regularly during the remainder of

8 "Ephynal Acetate," obtained through the courtesy of Dr. R. J. Floody, Hoffmann-La Roche, Inc., Nutley, N. J. the test. In a previous study (1) it was shown that the diets employed had no significant effect on the plasma tocopherol level.

Combined saturation, tolerance, and excretion study. Each of the subjects was studied for six consecutive threeday periods. The plasma tocopherol concentration was determined daily, while fecal analyses were carried out on pooled three-day collections marked off with orally administered carmine-red.

Oral tocopherol tolerance tests were carried out on day three, at the beginning of Period II, and again on day ten during Period IV. The first test followed a three-day control period of the diet alone. The second test followed the administration of $4000 \mathrm{mg}$. of dl-alpha-tocopheryl acetate $(3,656 \mathrm{mg}$. of free tocopherol) given as follows: day three $500 \mathrm{mg}$., days four through eight $200 \mathrm{mg}$. t.i.d., day nine none, and day ten $500 \mathrm{mg}$.

The following is a summary of the procedures carried out:

\begin{tabular}{|c|c|c|c|c|}
\hline Day & $\begin{array}{c}\text { Plasma } \\
\text { tocopherol }\end{array}$ & $\begin{array}{c}\text { Fecal } \\
\text { tocopherol }\end{array}$ & $\begin{array}{c}\text { Tocopheryl } \\
\text { acetate } \\
\text { administered } \\
\text { per period }\end{array}$ & $\begin{array}{l}\text { Tocopherol acetate } \\
\text { administered daily }\end{array}$ \\
\hline $\begin{array}{r}0 \\
1 \\
2 \\
3 \\
\\
4 \\
5 \\
6 \\
7 \\
8 \\
9 \\
10 \\
\\
11 \\
12 \\
13 \\
14 \\
15 \\
16 \\
17 \\
18\end{array}$ & $\left.\begin{array}{l} \\
* \\
* \\
* \\
* \\
* \\
* \\
* \\
* \\
* \\
* \\
* \\
* \\
* \\
* \\
* \\
* \\
*\end{array}\right\}$ & $\begin{array}{l}\text { Period I } \\
\text { Period II } \\
\text { Period III } \\
\text { Period IV } \\
\text { Period V } \\
\text { Period VI }\end{array}$ & $\begin{array}{r}500 \\
0 \\
0\end{array}$ & 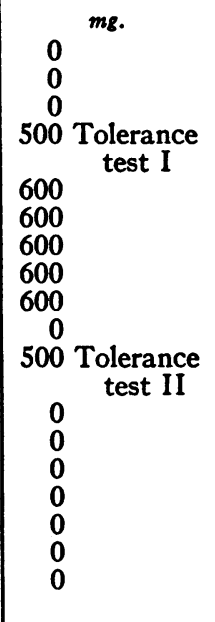 \\
\hline
\end{tabular}

In addition to these studies, complete urine collections were made daily for creatine and creatinine analysis, a sample of blood was tested for dialuric acid hemolysis, and, in the cirrhotic group, a section of liver obtained by needle biopsy was stained for acid-fast ceroid in an attempt to demonstrate physiological evidence of vitamin $\mathrm{E}$ deficiency. The results of these studies will be reported elsewhere.

\section{RESULTS}

Fasting plasma tocopherol level. The mean plasma tocopherol concentration was $1.22 \pm 0.35$ mg. per cent in the normal healthy adults, $0.92 \pm$ $0.32 \mathrm{mg}$. per cent in the cirrhotics, and $0.97 \pm 0.33$ mg. per cent in the convalescent controls. The concentration in the cirrhotic group was significantly lower than in the normal healthy adults, but 
TABLE I

Fasting plasma tocopherol concentration

\begin{tabular}{|c|c|c|c|c|c|c|}
\hline & $\begin{array}{l}\text { Number of } \\
\text { observations }\end{array}$ & $\begin{array}{l}\text { Number of } \\
\text { subjects }\end{array}$ & Maximum & Minimum & Mean & $\begin{array}{l}\text { Standard } \\
\text { deviation }\end{array}$ \\
\hline $\begin{array}{l}\text { Total observations* } \\
\text { normals } \\
\text { cirrhotics } \\
\text { convalescents }\end{array}$ & $\begin{array}{l}41 \\
69 \\
78\end{array}$ & $\begin{array}{l}31 \\
33 \\
60\end{array}$ & $\begin{array}{c}\text { me. per cent } \\
2.00 \\
1.70 \\
2.01\end{array}$ & $\begin{array}{c}\text { me. per cent } \\
0.62 \\
0.29 \\
0.35\end{array}$ & $\begin{array}{c}\text { me. per cent } \\
1.22 \\
0.92 \\
0.97\end{array}$ & $\begin{array}{l}\text { me. per cent } \\
\pm 0.35 \\
\pm 0.32 \\
\pm 0.33\end{array}$ \\
\hline $\begin{array}{l}\text { Individual observationst } \\
\text { (initial values only) } \\
\text { normals } \\
\text { cirrhotics } \\
\text { convalescents }\end{array}$ & $\begin{array}{l}31 \\
33 \\
60\end{array}$ & $\begin{array}{l}31 \\
33 \\
60\end{array}$ & $\begin{array}{l}2.00 \\
1.40 \\
2.01\end{array}$ & $\begin{array}{l}0.64 \\
0.29 \\
0.35\end{array}$ & $\begin{array}{l}1.22 \\
0.86 \\
0.99\end{array}$ & $\begin{array}{l} \pm 0.33 \\
\pm 0.29 \\
\pm 0.35\end{array}$ \\
\hline
\end{tabular}

* Mean value for normals significantly greater than that of cirrhotics $(t=3.80, p<0.01)$ and convalescents $(t=4.73, p>0.01)$. No significant difference between means for cirrhotics and convalescents $(t=0.88, p>0.20)$.

$\dagger$ Mean value for normals significantly greater than that of cirrhotics $(t=3.26, p<0.01)$ and convalescents $(t=4.62, p>0.01)$. No significant difference between means for cirrhotics and convalescents $(t=1.89, p>0.05)$.

TABLE II

Relation of fasting plasma tocopherol concentration to tocopherol administration

\begin{tabular}{|c|c|c|c|c|c|c|c|c|c|c|c|c|c|c|c|c|c|c|}
\hline Day & 1 & 2 & 3 & 4 & 5 & 6 & 7 & 8 & 9 & 10 & 11 & 12 & 13 & 14 & 15 & 16 & 17 & 18 \\
\hline $\begin{array}{c}\text { Tocopherol } \\
\text { administered (mg.) }\end{array}$ & 0 & 0 & 457 & 548 & 548 & 548 & 548 & 548 & 0 & 457 & 0 & 0 & 0 & 0 & 0 & 0 & 0 & 0 \\
\hline $\begin{array}{c}\text { Normals } \\
1 \\
2 \\
3 \\
4 \\
5 \\
6 \\
7 \\
8\end{array}$ & $\begin{array}{l}0.64 \\
0.90 \\
0.90 \\
1.06 \\
1.20 \\
1.37 \\
1.73 \\
1.75\end{array}$ & $\begin{array}{l}0.69 \\
0.90 \\
0.94 \\
1.12 \\
1.22 \\
1.34 \\
1.72 \\
1.72\end{array}$ & $\begin{array}{l}0.62 \\
0.89 \\
0.92 \\
1.09 \\
1.25 \\
1.36 \\
1.78 \\
1.74\end{array}$ & $\begin{array}{l}0.82 \\
1.56 \\
1.06 \\
1.58 \\
1.64 \\
1.64 \\
2.82 \\
2.12\end{array}$ & $\begin{array}{l}-\overline{-} \\
1.37 \\
1.68 \\
2.13 \\
1.68 \\
3.12 \\
2.21\end{array}$ & $\begin{array}{l}0.98 \\
1.70 \\
1.74 \\
2.26 \\
1.74 \\
3.25 \\
2.18\end{array}$ & $\begin{array}{l}1.15 \\
1.80 \\
1.69 \\
1.82 \\
- \\
1.80 \\
-\end{array}$ & $\begin{array}{l}1.04 \\
1.92 \\
1.63 \\
1.78 \\
2.36 \\
1.76 \\
3.02 \\
2.46\end{array}$ & $\begin{array}{l}1.15 \\
1.96 \\
2.12 \\
1.84 \\
2.34 \\
1.94 \\
3.13 \\
2.38\end{array}$ & $\begin{array}{l}1.04 \\
1.94 \\
1.67 \\
1.64 \\
1.86 \\
1.90 \\
2.86 \\
2.26\end{array}$ & $\begin{array}{l}0.64 \\
1.91 \\
1.48 \\
1.64 \\
1.68 \\
1.84 \\
2.08 \\
2.44\end{array}$ & $\begin{array}{l}- \\
1.24 \\
1.35 \\
1.48 \\
1.64 \\
1.86 \\
1.86\end{array}$ & $\begin{array}{l}0.85 \\
1.76 \\
1.37 \\
1.32 \\
1.53 \\
1.89 \\
1.74\end{array}$ & $\begin{array}{l}0.82 \\
1.68 \\
0.95 \\
1.28 \\
- \\
1.52 \\
-\end{array}$ & $\begin{array}{l}0.73 \\
1.40 \\
1.18 \\
1.20 \\
1.20 \\
1.42 \\
1.84 \\
1.78\end{array}$ & $\begin{array}{l}0.66 \\
1.18 \\
0.92 \\
1.14 \\
1.22 \\
1.38 \\
1.74 \\
1.70\end{array}$ & $\begin{array}{l}0.64 \\
0.92 \\
0.94 \\
1.10 \\
1.21 \\
1.34 \\
1.77 \\
1.76\end{array}$ & $\begin{array}{l}0.62 \\
0.89 \\
0.90 \\
1.08 \\
1.20 \\
1.30 \\
1.70 \\
1.74\end{array}$ \\
\hline Mean & 1.19 & 1.21 & 1.21 & 1.66 & 2.03 & 1.98 & 1.65 & 2.00 & 2.11 & 1.90 & 1.71 & 1.60 & 1.49 & 1.25 & 1.34 & 1.24 & 1.21 & 1.18 \\
\hline $\begin{array}{c}\text { Cirrhotics } \\
9 \\
10 \\
11 \\
12 \\
13 \\
14 \\
15 \\
16 \\
17 \\
18 \\
19\end{array}$ & $\begin{array}{l}0.34 \\
0.39 \\
0.54 \\
0.60 \\
0.92 \\
0.94 \\
1.05 \\
1.09 \\
1.10 \\
1.12 \\
1.36\end{array}$ & \begin{tabular}{l|} 
\\
0.52 \\
0.56 \\
0.52 \\
0.90 \\
0.89 \\
0.98 \\
1.10 \\
1.14 \\
1.16 \\
1.32
\end{tabular} & $\begin{array}{l}0.38 \\
0.42 \\
0.58 \\
0.61 \\
0.94 \\
0.86 \\
1.06 \\
1.01 \\
1.08 \\
1.09 \\
1.38\end{array}$ & $\begin{array}{l}0.44 \\
0.74 \\
0.85 \\
0.78 \\
1.91 \\
1.30 \\
- \\
1.94 \\
1.98 \\
1.41 \\
2.12\end{array}$ & $\begin{array}{l}0.51 \\
- \\
\bar{Z} \\
2.16 \\
1.51 \\
2.16 \\
2.05 \\
\overline{1.56} \\
2.00\end{array}$ & $\begin{array}{l}0.62 \\
1.09 \\
1.78 \\
1.95 \\
1.74 \\
2.06 \\
- \\
1.78 \\
1.80 \\
1.75\end{array}$ & $\begin{array}{l}\overline{1.03} \\
1.35 \\
2.48 \\
2.02 \\
1.86 \\
2.26 \\
2.70 \\
1.86 \\
1.89 \\
1.78\end{array}$ & $\begin{array}{l}-\overline{1.36} \\
1.36 \\
1.87 \\
2.08 \\
2.57 \\
2.34 \\
\overline{1.95} \\
1.78 \\
2.10\end{array}$ & $\begin{array}{l}1.28 \\
1.42 \\
1.16 \\
\overline{1.99} \\
- \\
\overline{2.80} \\
1.80 \\
-\end{array}$ & $\begin{array}{l}1.33 \\
1.34 \\
1.01 \\
1.46 \\
1.95 \\
1.90 \\
2.04 \\
2.58 \\
1.44 \\
1.37 \\
2.18\end{array}$ & $\begin{array}{l}1.17 \\
1.10 \\
1.09 \\
1.28 \\
1.56 \\
1.47 \\
1.64 \\
2.18 \\
1.84 \\
1.58 \\
1.86\end{array}$ & $\begin{array}{l}1.04 \\
0.90 \\
- \\
1.61 \\
2.15 \\
1.60 \\
2.11 \\
\overline{1.38} \\
1.64\end{array}$ & $\begin{array}{l}0.74 \\
-\overline{0} \\
0.93 \\
0.89 \\
1.58 \\
1.67 \\
1.54 \\
1.40 \\
1.30 \\
1.53\end{array}$ & $\begin{array}{l}0.80 \\
0.69 \\
1.04 \\
0.90 \\
-1.38 \\
1.48 \\
- \\
\overline{1.27} \\
1.46\end{array}$ & $\begin{array}{l}-\overline{-} \\
0.92 \\
1.02 \\
1.64 \\
1.24 \\
1.28 \\
1.94 \\
\overline{0.95} \\
1.34\end{array}$ & $\begin{array}{l}0.74 \\
0.55 \\
0.78 \\
0.96 \\
1.56 \\
- \\
- \\
1.71 \\
1.20 \\
-\end{array}$ & $\begin{array}{l}0.64 \\
0.54 \\
0.62 \\
0.94 \\
1.50 \\
1.36 \\
1.16 \\
1.56 \\
\overline{1.04} \\
1.36\end{array}$ & $\begin{array}{l}0.58 \\
0.56 \\
0.55 \\
0.92 \\
1.48 \\
1.38 \\
1.13 \\
1.52 \\
1.20 \\
1.14 \\
1.34\end{array}$ \\
\hline Mean & 0.86 & 0.91 & 0.85 & 1.35 & 1.71 & 1.62 & 1.92 & 1.93 & 1.74 & 1.69 & 1.53 & 1.55 & 1.29 & 1.13 & 1.29 & 1.07 & 1.07 & 1.07 \\
\hline $\begin{array}{l}\text { Convalescents } \\
20 \\
21 \\
22 \\
23 \\
24 \\
25 \\
26\end{array}$ & $\begin{array}{l}0.63 \\
0.71 \\
0.72 \\
0.76 \\
0.86 \\
0.98 \\
1.09\end{array}$ & $\begin{array}{l}0.62 \\
0.75 \\
0.72 \\
0.74 \\
0.87 \\
0.94 \\
1.02\end{array}$ & $\begin{array}{l}0.66 \\
0.72 \\
0.74 \\
0.78 \\
0.85 \\
1.05 \\
1.12\end{array}$ & $\begin{array}{l}0.74 \\
1.26 \\
0.76 \\
0.94 \\
0.91 \\
1.86 \\
1.84\end{array}$ & $\begin{array}{l}0.82 \\
1.38 \\
0.80 \\
1.02 \\
\overline{1.72} \\
1.98\end{array}$ & $\begin{array}{l}0.90 \\
1.44 \\
0.92 \\
1.14 \\
1.80 \\
1.95 \\
2.03\end{array}$ & $\begin{array}{l}0.98 \\
\overline{1.22} \\
1.22 \\
1.88 \\
\frac{-}{1.90}\end{array}$ & $\begin{array}{l}1.14 \\
1.46 \\
1.30 \\
- \\
2.18 \\
1.96\end{array}$ & $\begin{array}{l}1.20 \\
1.58 \\
- \\
2.13 \\
2.02 \\
1.86\end{array}$ & $\begin{array}{l}0.96 \\
1.40 \\
1.20 \\
1.20 \\
1.80 \\
2.11 \\
1.78\end{array}$ & $\begin{array}{l}0.94 \\
1.32 \\
1.18 \\
1.24 \\
1.80 \\
2.66 \\
1.69\end{array}$ & $\begin{array}{l}0.89 \\
1.14 \\
1.10 \\
1.12 \\
-2.40 \\
1.59\end{array}$ & $\begin{array}{l}0.90 \\
0.86 \\
0.94 \\
0.94 \\
- \\
\overline{1.46}\end{array}$ & $\begin{array}{l}\overline{0.82} \\
0.96 \\
0.86 \\
\overline{1.71} \\
-\end{array}$ & $\begin{array}{l}0.92 \\
0.72 \\
0.84 \\
- \\
1.14 \\
-\end{array}$ & $\begin{array}{l}0.90 \\
= \\
\overline{-} \\
1.64 \\
1.16\end{array}$ & $\begin{array}{l}0.94 \\
0.74 \\
0.77 \\
0.63 \\
0.85 \\
1.58 \\
-\end{array}$ & $\begin{array}{l}0.92 \\
0.70 \\
0.74 \\
0.78 \\
\overline{1.28} \\
1.10\end{array}$ \\
\hline Mean & 0.82 & 0.81 & 0.85 & 1.19 & 1.29 & 1.45 & 1.44 & 1.61 & 1.76 & 1.49 & 1.55 & 1.37 & 1.02 & 1.09 & 0.91 & 1.23 & 0.92 & 0.92 \\
\hline
\end{tabular}


did not differ significantly from that in the convalescent controls (Table I). However, it will be noted that there was considerable overlapping of values in all three groups, and that low values were by no means a constant finding in cirrhosis. Moreover, in the cirrhotic group there was no correlation between the plasma level and the state of hepatic function, as judged by the serum concentrations of albumin $(n=27, r=+0.330, p>$ $0.05)$ and bilirubin $(n=41, r=-0.043, p>$ $0.05)$.

These observations suggested that extrahepatic factors might be more important in determining the plasma tocopherol level in cirrhosis than the state of hepatic function.

Effect of tissue saturation on the plasma tocopherol level. An attempt was made to saturate the tissues with tocopherol to determine whether low plasma levels were indicative of relatively depleted tissue stores. The amount of tocopherol administered, 3.656 grams in eight days, exceeded the estimated total body content (8), so that appreciable tissue saturation probably occurred, even though simultaneous fecal studies revealed that only 60 to 70 per cent was absorbed.
During the administration of tocopherol the plasma level rose steadily, usually reaching a peak on the fifth and sixth day (Table II). At the peak neither the absolute values attained nor the increases above the initial levels differed significantly in the three groups of subjects (Table III). However, following the withdrawal of tocopherol, there was a prompt fall to the pretreatment level in the normal group, while the level remained elevated in eight of the 11 cirrhotics and two of the seven convalescent controls (Table II). The mean increase in concentration eight days following cessation of tocopherol administration was statistically significant in the cirrhotic group, but not in the convalescent controls (Table III).

The readiness with which the plasma concentration could be raised by means of orally administered tocopherol and the sustained character of the increase following presumed saturation of the tissues suggested that the low plasma levels in cirrhosis were probably more closely related to tissue depletion than to any impairment of absorption or utilization. A similar sustained increase in plasma tocopherol was observed following the administration of tocopherol to individuals

TABLE III

Effect of daily tocopherol administration on fasting plasma tocopherol concentration

\begin{tabular}{|c|c|c|c|c|c|}
\hline & $\underbrace{\text { Number of }}_{\text {subjects }}$ & Maximum & Minimum & Mean & $\begin{array}{l}\text { Standard } \\
\text { deviation }\end{array}$ \\
\hline $\begin{array}{l}\text { Maximum increase in plasma tocopherol } \\
\text { over control level, mg. per cent } \\
\text { normals } \\
\text { cirrhotics } \\
\text { convalescents }\end{array}$ & $\begin{array}{r}8 \\
11 \\
7\end{array}$ & $\begin{array}{l}1.20 \\
1.90 \\
1.67\end{array}$ & $\begin{array}{l}0.50 \\
0.67 \\
0.48\end{array}$ & $\begin{array}{l}0.93^{*} \\
1.17^{*} \\
0.91^{*}\end{array}$ & $\begin{array}{l} \pm 0.33 \\
\pm 0.40 \\
\pm 0.41\end{array}$ \\
\hline $\begin{array}{l}\text { Maximum concentration of plasma toco- } \\
\text { pherol attained, mg. per cent } \\
\text { normals } \\
\text { cirrhotics } \\
\text { convalescents }\end{array}$ & $\begin{array}{r}8 \\
11 \\
7\end{array}$ & $\begin{array}{l}3.25 \\
2.80 \\
2.66\end{array}$ & $\begin{array}{l}1.15 \\
1.33 \\
1.20\end{array}$ & $\begin{array}{l}2.14^{*} \\
2.05^{*} \\
1.73^{*}\end{array}$ & $\begin{array}{l} \pm 0.56 \\
\pm 0.48 \\
\pm 0.52\end{array}$ \\
\hline $\begin{array}{l}\text { Day of tocopherol administration on which } \\
\text { maximum concentration was attained } \\
\text { normals } \\
\text { cirrhotics } \\
\text { convalescents }\end{array}$ & $\begin{array}{r}8 \\
11 \\
7\end{array}$ & $\begin{array}{l}6 \\
7 \\
8\end{array}$ & $\begin{array}{l}3 \\
4 \\
3\end{array}$ & $\begin{array}{l}5.1^{*} \\
5.4^{*} \\
6.0^{*}\end{array}$ & $\begin{array}{l} \pm 1.2 \\
\pm 0.8 \\
\pm 1.6\end{array}$ \\
\hline $\begin{array}{l}\text { Difference between tocopherol concentra- } \\
\text { tion } 8 \text { days following cessation of to- } \\
\text { copherol administration and initial } \\
\text { control level } \\
\text { normals } \\
\text { cirrhotics } \\
\text { convalescents }\end{array}$ & $\begin{array}{r}8 \\
11 \\
7\end{array}$ & $\begin{array}{r}0.00 \\
+0.56 \\
+0.29\end{array}$ & $\begin{array}{l}-0.06 \\
-0.01 \\
-0.03\end{array}$ & $\begin{array}{l}-0.02 \uparrow \\
+0.21 \\
+0.08\end{array}$ & $\begin{array}{l} \pm 0.02 \\
\pm 0.20 \\
\pm 0.13\end{array}$ \\
\hline
\end{tabular}

* Difference between means not statistically significant.

+ Difference between normals and cirrhotics highly significant $(t=3.71, p<0.01)$; difference between normals and convalescents, or cirrhotics and convalescents not statistically significant. 
TABLE IV

Results of oral tocopherol tolerance tests (500 mg. dl-alpha-tocopheryl acetate)

\begin{tabular}{|c|c|c|c|c|c|}
\hline & $\begin{array}{l}\text { Number of } \\
\text { subjects }\end{array}$ & Maximum & Minimum & Mean & $\begin{array}{l}\text { Standard } \\
\text { deviation }\end{array}$ \\
\hline $\begin{array}{l}\text { Maximum increase in plasma tocopherol } \\
\text { concentration, mg. per cent } \\
\text { normals } \\
\text { cirrhotics } \\
\text { convalescents }\end{array}$ & $\begin{array}{r}8 \\
26 \\
28\end{array}$ & $\begin{array}{l}2.00 \\
1.86 \\
3.25\end{array}$ & $\begin{array}{l}0.20 \\
0.00 \\
0.12\end{array}$ & $\begin{array}{l}0.83 \\
0.70^{*} \\
1.12^{*}\end{array}$ & $\begin{array}{l} \pm 0.57 \\
\pm 0.12 \\
\pm 0.85\end{array}$ \\
\hline $\begin{array}{l}\text { Mean increase in plasma tocopherol con- } \\
\text { centration, mg. per cent (calculated } \\
\text { from area of curves) } \\
\text { normals } \\
\text { cirrhotics } \\
\text { convalescents }\end{array}$ & $\begin{array}{r}8 \\
26 \\
28\end{array}$ & $\begin{array}{l}0.89 \\
1.17 \\
1.96\end{array}$ & $\begin{array}{l}0.13 \\
0.00 \\
0.00\end{array}$ & $\begin{array}{l}0.48 \dagger \\
0.39 \\
0.57 \dagger\end{array}$ & $\begin{array}{l} \pm 0.27 \\
\pm 0.29 \\
\pm 0.51\end{array}$ \\
\hline $\begin{array}{l}\text { Maximum concentration of plasma toco- } \\
\text { pherol during tolerance test, mg. per } \\
\text { cent } \\
\text { normals } \\
\text { cirrhotics } \\
\text { convalescents }\end{array}$ & $\begin{array}{r}8 \\
26 \\
28\end{array}$ & $\begin{array}{l}3.09 \\
2.64 \\
4.98\end{array}$ & $\begin{array}{l}0.82 \\
0.56 \\
0.76\end{array}$ & $\begin{array}{l}2.04 t \\
1.65 t \\
2.03 t\end{array}$ & $\begin{array}{l} \pm 0.76 \\
\pm 0.60 \\
\pm 0.99\end{array}$ \\
\hline
\end{tabular}

* Difference between cirrhotics and convalescents statistically significant $(t=2.59, p<0.02)$; difference between cirrhotics and normals, or convalescents and normals not statistically significant.

† Difference between means not statistically significant.

whose previous intake had been low and whose tissues had presumably been depleted (9). Judging from the results of animal feeding experiments (10) it may be presumed that the tissue stores were likewise increased by tocopherol administration in the normal and convalescent controls, even though there was no sustained increase in plasma concentration. These findings suggest that there may be mechanisms tending to stabilize the plasma tocopherol level within the normal range.
Fractionation of plasma tocopherol. Since tocopheryl esters fail to react in the Emmerie-Engel reaction, on which our analytical procedures were based, they would go undetected leading to falsely low values. It was necessary, therefore, to exclude their presence by analyzing the plasma following saponification. By this method, no tocopheryl esters could be demonstrated in the plasma, even after large doses of tocopheryl acetate were administered. Thus, in a cirrhotic with

TABLE $v$

Comparison of oral tocopherol tolerance tests before and after the administration of 3.5 grams of dl-alpha-tocopheryl acetate over a six-day period

\begin{tabular}{|c|c|c|c|c|c|c|c|c|c|c|c|c|c|}
\hline & \multirow{2}{*}{$\begin{array}{l}\text { Number } \\
\text { of } \\
\text { subjects }\end{array}$} & \multicolumn{4}{|c|}{$\begin{array}{l}\text { Maximum increase in plasma } \\
\text { tocopherol concentration }\end{array}$} & \multicolumn{4}{|c|}{$\begin{array}{l}\text { Mean rise in plasma } \\
\text { tocopherol concentration }\end{array}$} & \multicolumn{4}{|c|}{$\begin{array}{l}\text { Maximum plasma tocopherol } \\
\text { at height of curve }\end{array}$} \\
\hline & & $\underset{\text { mumi- }}{\text { Maxi- }}$ & $\begin{array}{l}\text { Mini- } \\
\text { mum }\end{array}$ & Mean & $\begin{array}{l}\text { Standard } \\
\text { deviation }\end{array}$ & $\underset{\text { mumi- }}{\text { Maxi- }}$ & $\begin{array}{l}\text { Mini- } \\
\text { mum }\end{array}$ & Mean & $\begin{array}{l}\text { Standard } \\
\text { deviation }\end{array}$ & $\underset{\text { mumi- }}{\text { Maxi }}$ & $\begin{array}{l}\text { Mini- } \\
\text { mum }\end{array}$ & Mean & $\begin{array}{l}\text { Standard } \\
\text { deviation }\end{array}$ \\
\hline & & \multicolumn{4}{|c|}{ ms. per cent } & \multicolumn{4}{|c|}{ mg. per cent } & \multicolumn{4}{|c|}{ mg. per cent } \\
\hline $\begin{array}{l}\text { Normals } \\
\text { before } \\
\text { after }\end{array}$ & 8 & $\begin{array}{l}2.00 \\
1.32\end{array}$ & $\begin{array}{l}0.20 \\
0.06\end{array}$ & $\begin{array}{l}0.83^{*} \\
0.58^{*}\end{array}$ & $\begin{array}{l} \pm 0.57 \\
\pm 0.38\end{array}$ & $\begin{array}{l}0.89 \\
0.36\end{array}$ & $\begin{array}{l}0.13 \\
0.01\end{array}$ & $\begin{array}{l}0.48 t \\
0.20 \dagger\end{array}$ & $\begin{array}{l} \pm 0.27 \\
\pm 0.11\end{array}$ & $\begin{array}{l}3.09 \\
3.75\end{array}$ & $\begin{array}{l}0.82 \\
1.27\end{array}$ & $\begin{array}{l}2.04^{*} \\
2.48^{*}\end{array}$ & $\begin{array}{l} \pm 0.76 \\
\pm 0.70\end{array}$ \\
\hline $\begin{array}{l}\text { Cirrhotics } \\
\text { before } \\
\text { after }\end{array}$ & 11 & $\begin{array}{l}1.42 \\
1.70\end{array}$ & $\begin{array}{l}0.38 \\
0.00\end{array}$ & $\begin{array}{l}0.76^{*} \\
0.61^{*}\end{array}$ & $\begin{array}{l} \pm 0.37 \\
\pm 0.34\end{array}$ & $\begin{array}{l}0.87 \\
0.70\end{array}$ & $\begin{array}{l}0.16 \\
0.00\end{array}$ & $\begin{array}{l}0.44 \dagger \\
0.18 \dagger\end{array}$ & $\begin{array}{l} \pm 0.24 \\
\pm 0.18\end{array}$ & $\begin{array}{l}2.78 \\
3.14\end{array}$ & $\begin{array}{l}0.76 \\
1.46\end{array}$ & $\begin{array}{l}1.62 \dagger \\
2.28 \dagger\end{array}$ & $\begin{array}{l} \pm 0.62 \\
\pm 0.65\end{array}$ \\
\hline $\begin{array}{l}\text { Convalescents } \\
\text { before } \\
\text { after }\end{array}$ & 7 & $\begin{array}{l}1.12 \\
1.06\end{array}$ & $\begin{array}{l}0.12 \\
0.22\end{array}$ & $\begin{array}{l}0.57^{*} \\
0.67^{*}\end{array}$ & $\begin{array}{l} \pm 0.39 \\
\pm 0.08\end{array}$ & $\begin{array}{l}0.64 \\
0.41\end{array}$ & $\begin{array}{l}0.01 \\
0.06\end{array}$ & $\begin{array}{l}0.33^{*} \\
0.19^{*}\end{array}$ & $\begin{array}{l} \pm 0.24 \\
\pm 0.10\end{array}$ & $\begin{array}{l}2.14 \\
2.84\end{array}$ & $\begin{array}{l}0.76 \\
1.42\end{array}$ & $\begin{array}{l}1.40 \dagger \\
2.16 \dagger\end{array}$ & $\begin{array}{l} \pm 0.49 \\
\pm 0.58\end{array}$ \\
\hline
\end{tabular}

* Difference between means not statistically significant.

$\dagger$ Difference between means statistically significant: mean rise in normals, $t=2.56, p<0.05 ;$ in cirrhotics, $t=2.76$, $\mathrm{p}<0.02$; maximum plasma tocopherol in cirrhotics, $t=2.35, \mathrm{p}<0.05$; in convalescents, $t=2.45, \mathrm{p}<0.05$. 
low plasma levels of $0.37,0.42$, and $0.39 \mathrm{mg}$. per cent on successive days while receiving $600 \mathrm{mg}$. of dl-alpha tocopheryl acetate daily, there were recoveries of only $0.20,0.28$, and $0.22 \mathrm{mg}$. per cent following saponification. Similarly in a normal subject with a high plasma level of $2.78 \mathrm{mg}$. per cent during a similar period of tocopheryl acetate administration, only $1.65 \mathrm{mg}$. per cent could be recovered following saponification. These recoveries, representing 54 to 67 per cent of the original tocopherol present, were even smaller than those obtained following saponification of comparable concentrations of pure d-alpha-tocopherol in alcohol.

Other investigators $(11,12)$ have likewise failed to demonstrate the presence of tocopheryl esters in the plasma of normal animals following the oral administration of tocopheryl acetate or phosphate.

Oral tocopherol tolerance tests. To further investigate the possibility that the low plasma tocopherol levels in cirrhosis were more closely related to depleted tissue stores than to impaired absorption or hepatic dysfunction, the response of the plasma level to a single test dose of tocopheryl acetate was studied both before and after tissue saturation.

Before saturation, the cirrhotics showed a smaller rise in plasma tocopherol concentration than either the normal or convalescent controls (Table IV). However, the difference between the cirrhotic and normal subjects was not statistically significant, and when the tolerance curves were compared on the basis of the mean rise in concentration, calculated from the areas under the

TABLE VI

Relation of fecal tocopherol excretion to tocopherol intake

\begin{tabular}{|c|c|c|c|c|c|c|c|c|}
\hline & \multirow{2}{*}{$\begin{array}{l}\text { Number of } \\
\text { subjects }\end{array}$} & \multicolumn{3}{|c|}{ Tocopherol intake } & \multicolumn{4}{|c|}{ Fecal tocopherol excretion } \\
\hline & & Dietary & $\begin{array}{l}\text { Fed as } \\
\text { acetate }\end{array}$ & Total & Maximum & Minimum & Mean & $\begin{array}{l}\text { Standard } \\
\text { deviation }\end{array}$ \\
\hline & & \multicolumn{3}{|c|}{ me. per 3-day period } & \multicolumn{4}{|c|}{ mg. per 3-dey period } \\
\hline $\begin{array}{l}\text { Period I } \\
\quad \text { normals } \\
\text { cirrhotics } \\
\text { convalescents }\end{array}$ & $\begin{array}{l}6 \\
6 \\
5\end{array}$ & $\begin{array}{r}100.6 \\
63.1 \\
92.3\end{array}$ & $\begin{array}{l}0 \\
0 \\
0\end{array}$ & $\begin{array}{r}100.6 \\
63.1 \\
92.1\end{array}$ & $\begin{array}{l}74.37 \\
35.21 \\
62.58\end{array}$ & $\begin{array}{l}48.39 \\
12.26 \\
36.65\end{array}$ & $\begin{array}{l}64.85 \\
22.23^{*} \\
49.29 \dagger\end{array}$ & $\begin{array}{ll} \pm & 8.79 \\
\pm & 8.96 \\
\pm & 8.44\end{array}$ \\
\hline $\begin{array}{l}\text { Period II } \\
\text { normals } \\
\text { cirrhotics } \\
\text { convalescents }\end{array}$ & $\begin{array}{l}6 \\
6 \\
5\end{array}$ & $\begin{array}{r}100.6 \\
63.1 \\
92.3\end{array}$ & $\begin{array}{l}1,553.8 \\
1,553.8 \\
1,553.8\end{array}$ & $\begin{array}{l}1,654.4 \\
1,616.9 \\
1,646.1\end{array}$ & $\begin{array}{r}1,138.80 \\
644.49 \\
982.68\end{array}$ & $\begin{array}{l}797.04 \\
292.69 \\
742.73\end{array}$ & $\begin{array}{l}929.36 \\
507.65^{*} \\
861.54\end{array}$ & $\begin{array}{l} \pm 108.4 \\
\pm 119.6 \\
\pm 79.0\end{array}$ \\
\hline $\begin{array}{l}\text { Period III } \\
\text { normals } \\
\text { cirrhotics } \\
\text { convalescents }\end{array}$ & $\begin{array}{l}6 \\
6 \\
5\end{array}$ & $\begin{array}{r}100.6 \\
63.1 \\
92.3\end{array}$ & $\begin{array}{l}1,645.2 \\
1,645.2 \\
1,645.2\end{array}$ & $\begin{array}{l}1,745.8 \\
1,708.3 \\
1,737.5\end{array}$ & $\begin{array}{r}1,248.56 \\
840.09 \\
1,240.83\end{array}$ & $\begin{array}{l}969.15 \\
337.34 \\
950.10\end{array}$ & $\begin{array}{c}1,070.79 \\
634.85^{*} \\
1,080.24\end{array}$ & $\begin{array}{l} \pm 98.8 \\
\pm 157.6 \\
\pm 105.9\end{array}$ \\
\hline $\begin{array}{l}\text { Period IV } \\
\text { normals } \\
\text { cirrhotics } \\
\text { convalescents }\end{array}$ & $\begin{array}{l}6 \\
6 \\
5\end{array}$ & $\begin{array}{r}100.6 \\
63.1 \\
92.3\end{array}$ & $\begin{array}{l}457.0 \\
457.0 \\
457.0\end{array}$ & $\begin{array}{l}557.6 \\
520.1 \\
549.3\end{array}$ & $\begin{array}{l}429.57 \\
193.44 \\
286.08\end{array}$ & $\begin{array}{l}275.10 \\
126.99 \\
212.59\end{array}$ & $\begin{array}{l}330.22 \\
158.83^{*} \\
252.71\end{array}$ & $\begin{array}{l} \pm 49.5 \\
\pm 24.7 \\
\pm 24.6\end{array}$ \\
\hline $\begin{array}{l}\text { Period V } \\
\text { normals } \\
\text { cirrhotics } \\
\text { convalescents }\end{array}$ & $\begin{array}{l}6 \\
6 \\
5\end{array}$ & $\begin{array}{r}100.6 \\
63.1 \\
92.3\end{array}$ & $\begin{array}{l}\mathbf{0} \\
\mathbf{0} \\
\mathbf{0}\end{array}$ & $\begin{array}{r}\cdot \\
100.6 \\
63.1 \\
92.3\end{array}$ & $\begin{array}{r}100.31 \\
62.27 \\
84.33\end{array}$ & $\begin{array}{l}57.99 \\
36.90 \\
53.75\end{array}$ & $\begin{array}{l}79.97 \\
47.38^{*} \\
68.12\end{array}$ & $\begin{array}{l} \pm 14.70 \\
\pm \quad 8.42 \\
\pm 10.94\end{array}$ \\
\hline $\begin{array}{l}\text { Period VI } 8 \\
\text { normals } \\
\text { cirrhotics } \\
\text { convalescents }\end{array}$ & $\begin{array}{l}6 \\
6 \\
5\end{array}$ & $\begin{array}{r}100.6 \\
63.1 \\
92.3\end{array}$ & $\begin{array}{l}0 \\
0 \\
0\end{array}$ & $\begin{array}{r}100.6 \\
63.1 \\
92.3\end{array}$ & $\begin{array}{l}70.48 \\
48.39 \\
75.25\end{array}$ & $\begin{array}{l}54.78 \\
34.53 \\
45.71\end{array}$ & $\begin{array}{l}63.25 \\
38.53^{*} \\
60.08\end{array}$ & $\begin{array}{l} \pm \quad 5.32 \\
\pm \quad 5.03 \\
\pm \quad 9.70\end{array}$ \\
\hline
\end{tabular}

* Excretion in cirrhotics significantly lower than in normals and convalescents (Period $I, t=7.61$ and 4.66 , respectively; Period II, $t=5.86$ and 5.32; Period III, $t=5.25$ and 5.06; Period IV, $t=8.06$ and 5.68; Period V, $t=4.18$ and 3.00; Period VI, $t=7.14$ and 3.94; " $t$ " value of $3.17=p<0.01 ; t, 3.00=p<0.02$ ).

Excretion in convalescents significantly lower than in normals during Period I only $(t=2.71, p<0.05)$.

Excretion during Period V significantly greater than in Period I in convalescents and in cirrhotics ( $t=2.72$, $p<0.05$ and $t=4.33, p<0.01$, respectively). Difference between Periods $I$ and $V$ in normals of borderline significance $(t=1.98, p<0.10,>0.05)$.

8 Excretion during Period VI significantly greater than in Period I in cirrhotic group only $(t=2.38, p<0.05)$. 
TABLE VII

Percentage of ingested tocopherol recovered in the feces

\begin{tabular}{|c|c|c|c|c|c|c|}
\hline & \multirow{2}{*}{$\underset{\text { subjects }}{\text { Number of }}$} & \multirow{2}{*}{$\begin{array}{l}\text { Mg. tocopherol } \\
\text { ingested per } \\
\text { period }\end{array}$} & \multicolumn{4}{|c|}{ Per cent tocopherol recovered in feces } \\
\hline & & & Maximum & Minimum & Mean & $\begin{array}{l}\text { Standard } \\
\text { deviation }\end{array}$ \\
\hline $\begin{array}{l}\text { Period I } \\
\text { normals } \\
\text { cirrhotics } \\
\text { convalescents }\end{array}$ & $\begin{array}{l}6 \\
6 \\
5\end{array}$ & $\begin{array}{r}100.6 \\
63.1 \\
92.1\end{array}$ & $\begin{array}{l}73.9 \\
55.8 \\
67.9\end{array}$ & $\begin{array}{l}48.1 \\
19.4 \\
39.8\end{array}$ & $\begin{array}{l}64.4 \\
35.2 \dagger \\
53.5\end{array}$ & $\begin{array}{l} \pm 9.2 \\
\pm 14.2 \\
\pm 9.0\end{array}$ \\
\hline $\begin{array}{l}\text { Period II } \\
\text { normals } \\
\text { cirrhotics } \\
\text { convalescents }\end{array}$ & $\begin{array}{l}6 \\
6 \\
5\end{array}$ & $\begin{array}{l}1,654.4 \\
1,616.9 \\
1,646.1\end{array}$ & $\begin{array}{l}68.8 \\
39.9 \\
59.7\end{array}$ & $\begin{array}{l}48.2 \\
18.1 \\
45.1\end{array}$ & $\begin{array}{l}56.2 \\
31.4 \dagger \\
52.3\end{array}$ & $\begin{array}{l} \pm 6.4 \\
\pm 7.5 \\
\pm \quad 5.0\end{array}$ \\
\hline $\begin{array}{l}\text { Period III } \\
\text { normals } \\
\text { cirrhotics } \\
\text { convalescents }\end{array}$ & $\begin{array}{l}6 \\
6 \\
5\end{array}$ & $\begin{array}{l}1,745.8 \\
1,708.3 \\
1,737.5\end{array}$ & $\begin{array}{l}71.5 \\
49.2 \\
71.4\end{array}$ & $\begin{array}{l}55.5 \\
19.7 \\
54.7\end{array}$ & $\begin{array}{l}61.3 \\
37.2 \dagger \\
62.2\end{array}$ & $\begin{array}{l} \pm 6.0 \\
\pm 9.0 \\
\pm 5.9\end{array}$ \\
\hline $\begin{array}{l}\text { Period IV } \\
\text { normals } \\
\text { cirrhotics } \\
\text { convalescents }\end{array}$ & $\begin{array}{l}6 \\
6 \\
5\end{array}$ & $\begin{array}{l}557.6 \\
520.1 \\
549.3\end{array}$ & $\begin{array}{l}77.0 \\
37.2 \\
52.1\end{array}$ & $\begin{array}{l}49.3 \\
24.4 \\
38.7\end{array}$ & $\begin{array}{l}59.2 \\
30.6 \dagger \\
46.0 \ddagger\end{array}$ & $\begin{array}{l} \pm 9.0 \\
\pm 4.4 \\
\pm 4.5\end{array}$ \\
\hline $\begin{array}{l}\text { Period V\$ } \\
\text { normals } \\
\text { cirrhotics } \\
\text { convalescents }\end{array}$ & $\begin{array}{l}6 \\
6 \\
5\end{array}$ & $\begin{array}{r}100.6 \\
63.1 \\
92.3\end{array}$ & $\begin{array}{l}99.7 \\
98.7 \\
91.6\end{array}$ & $\begin{array}{l}57.6 \\
58.5 \\
58.4\end{array}$ & $\begin{array}{l}79.5 \\
75.1 \\
74.0\end{array}$ & $\begin{array}{l} \pm 14.6 \\
\pm 13.2 \\
\pm 11.7\end{array}$ \\
\hline $\begin{array}{l}\text { Period VI\& } \\
\text { normals } \\
\text { cirrhotics } \\
\text { convalescents }\end{array}$ & $\begin{array}{l}6 \\
6 \\
5\end{array}$ & $\begin{array}{r}100.6 \\
63.1 \\
92.3\end{array}$ & $\begin{array}{l}70.1 \\
76.7 \\
81.7\end{array}$ & $\begin{array}{r}54.5 \\
54.7 \\
49.6\end{array}$ & $\begin{array}{l}62.9 \\
61.1 \\
65.2\end{array}$ & $\begin{array}{l} \pm 4.9 \\
\pm 7.7 \\
\pm 10.6\end{array}$ \\
\hline
\end{tabular}

* Calculated content of diet plus tocopherol administered as acetate (see Table VI).

$\dagger$ Excretion in cirrhotics significantly less than in normals or convalescents during Periods I through IV (Period I, $t=3.89$ and 2.36, respectively; Period II, $t=5.63$ and 5.06; Period III, $t=4.98$ and 5.06; Period $I V, t=6.39$ and 5.18; " $t$ " value of $3.17=\mathrm{p}<0.01$ ).

\pm Excretion in convalescents significantly less than in normals during Period IV only $(t=2.88, p<0.02)$.

Excretion during Period V significantly greater than in Period $I$ in cirrhotics $(t=4.36, p<0.01)$ and convalescents $(t=2.75, p<0.05)$; difference of borderline significance in normals $(t=1.96, p<0.10>0.05)$; excretion during Period VI significantly greater than in Period $I$ in cirrhotics only $(t=3.51, p<0.01)$.

curves measured with a planimeter, or on the basis of the maximum absolute values attained at the peak, no significant differences were discerned between any of the groups. Moreover, in the cirrhotic group no significant correlations could be demonstrated between the magnitude of the rise in plasma tocopherol concentration and the initial level $(n=25, r=+0.274, p>0.05)$, or the state of hepatic function, as judged by the concentrations of serum albumin $(n=19, r=-0.160$, $p>0.05)$ and bilirubin $(n=25, r=-0.080$, $\mathrm{p}>0.05$ ).

Following saturation of the tissues with tocopherol, the tolerance curves in the three groups no longer differed, whether compared on the basis of the maximum or mean rise in plasma tocopherol, or on the basis of the absolute values attained at the peak of the curves (Table V). Unfortunately, in the three small groups of subjects, randomlyselected for testing before and after saturation, there were no differences between the tolerance curves before saturation (Table V), so that the uniformity of the curves observed after saturation could not be ascribed to a change in the state of the tissue stores.

When the tolerance tests carried out before and after saturation were compared (Table V), it was found that the rise in plasma tocopherol concentration was smaller after saturation than before, both in the normal and cirrhotic groups. The differences were not statistically significant when the comparison was based on the maximum increase in plasma tocopherol, but were significant when based on the mean rise calculated from the 
TABLE VIII

Correlations between fecal excretion of tocopherol and the plasma tocopherol level, the results of the tocopherol tolerance test and the state of hepatic function

\begin{tabular}{|c|c|c|c|c|c|c|}
\hline \multirow{2}{*}{ Subject } & \multirow{2}{*}{$\begin{array}{l}\text { Fecal tocopherol, } \\
\text { Period } I^{*}\end{array}$} & \multirow{2}{*}{$\begin{array}{c}\text { Mean fasting } \\
\text { plasma } \\
\text { tocopherol* }\end{array}$} & \multicolumn{2}{|c|}{ Tocopherol tolerance test } & \multirow{2}{*}{$\begin{array}{l}\text { Serum } \\
\text { bilirubin }\end{array}$} & \multirow{2}{*}{$\begin{array}{l}\text { Serum } \\
\text { albumin }\end{array}$} \\
\hline & & & Maximum rise & Mean rise & & \\
\hline $\begin{array}{l}\text { Cirrhotic } \\
\text { Cirrhotic } \\
\text { Cirrhotic } \\
\text { Cirrhotic } \\
\text { Cirrhotic } \\
\text { Cirrhotic } \\
\text { Convalescent } \\
\text { Convalescent } \\
\text { Convalescent } \\
\text { Normal } \\
\text { Convalescent } \\
\text { Normal } \\
\text { Convalescent } \\
\text { Normal } \\
\text { Normal } \\
\text { Normal } \\
\text { Normal }\end{array}$ & $\begin{array}{c}\text { mg. per 3-day } \\
\text { period } \\
12.26 \\
12.51 \\
18.37 \\
22.62 \\
32.42 \\
35.21 \\
36.65 \\
46.64 \\
48.03 \\
48.39 \\
52.54 \\
59.93 \\
62.58 \\
64.51 \\
69.81 \\
72.09 \\
74.37\end{array}$ & $\begin{array}{c}\text { ms. per cent } \\
0.36 \\
1.11 \\
0.90 \\
0.58 \\
1.03 \\
0.44 \\
0.73 \\
0.76 \\
0.64 \\
1.09 \\
0.86 \\
1.22 \\
0.73 \\
0.65 \\
1.74 \\
1.74 \\
0.92\end{array}$ & $\begin{array}{c}\text { me. per cent } \\
0.38 \\
0.90 \\
0.44 \\
0.45 \\
1.42 \\
0.54 \\
0.02 \\
0.42 \\
0.60 \\
2.00 \\
0.12 \\
0.43 \\
0.70 \\
0.20 \\
1.11 \\
0.90 \\
0.23\end{array}$ & $\begin{array}{c}\text { mg. per cent } \\
0.21 \\
0.60 \\
0.16 \\
0.28 \\
0.38 \\
0.35 \\
0.01 \\
0.22 \\
0.22 \\
0.81 \\
0.09 \\
0.28 \\
0.51 \\
0.15 \\
0.89 \\
0.50 \\
0.13\end{array}$ & $\begin{array}{c}\text { ms. per cent } \\
6.82 \\
1.10 \\
1.58 \\
2.72 \\
12.46 \\
3.58 \\
0.74 \\
0.60 \\
0.82 \\
0.48 \\
0.64 \\
0.84 \\
0.46 \\
0.60 \\
0.74\end{array}$ & $\begin{array}{c}\text { em. per cent } \\
1.71 \\
2.70 \\
2.20 \\
2.39 \\
1.79 \\
1.48\end{array}$ \\
\hline
\end{tabular}

* Coefficient of correlation $r=+0.488, p<0.05$.

areas under the curves. However, as might be expected from the higher plasma concentrations at the start of the post-saturation test, the maximum absolute values attained at the peak of the curves were significantly higher than before saturation.

It is difficult to account for the smaller rise in plasma tocopherol in the post-saturation tolerance test, unless it is assumed that there was an upper limit of concentration which could not be exceeded. If there was such a ceiling it varied in different individuals, since the maximum values observed ranged from 1.27 to $3.75 \mathrm{mg}$. per cent (Table V), and could have been determined by several factors, including the relative rates of tocopherol absorption in the intestine and deposition in the tissues, the rate of excretion in the bile or urine, and possibly, the capacity of the plasma to transport tocopherol.

The results of the tolerance tests must be regarded as inconclusive with respect to the problem of whether the low plasma tocopherol levels found in cirrhosis are related to impaired absorption, or to unsaturated tissue stores dependent on other factors.

Fecal excretion of tocopherol. To investigate the factor of absorption more directly, studies of fecal tocopherol excretion were undertaken. These revealed that the cirrhotics excreted less tocopherol than either the normal or convalescent con- trols, both during the control period and following the administration of tocopheryl acetate (Table VI). The convalescents also excreted less tocopherol than the normals, but the difference was not statistically significant, except during the control period.

The amount of tocopherol excreted was roughly proportional to the intake (Table VI), but it is evident from the data in Table VII that the proportion excreted in the cirrhotic group was significantly lower than in the normal or convalescent controls, both during the control period and during tocopherol administration. The convalescents also excreted a smaller fraction of ingested tocopherol than the normal controls, but the difference was not statistically significant, except in $\mathrm{Pe}-$ riod IV (Table VII).

Immediately following the cessation of tocopherol administration there was a sharp fall in fecal tocopherol excretion, but it was still significantly greater in Period V than in Period I in all groups (Table VI), so that the ratios of excreted to ingested tocopherol rose to unusually high levels (Table VII). In the succeeding period, however, the normal and convalescent subjects returned to their control levels, but the cirrhotics did not, so that in Period VI the proportion of ingested tocopherol excreted no longer differed in the three groups (Table VII). 
There appeared to be a significant relationship between the tocopherol content of the feces during the control period and its concentration in the plasma, but no correlations could be demonstrated between fecal tocopherol and the state of hepatic function or the results of the tocopherol tolerance tests (Table VIII).

These findings strongly suggested that there was no impairment of absorption in the cirrhotics, and that, actually, when large doses of tocopherol were ingested the proportion absorbed was even greater than in the normal or convalescent controls. However, the validity of estimating tocopherol absorption from the results of fecal analyses still had to be established. In particular it was necessary to exclude the presence of esterified tocopherol in the feces, and to rule out the occurrence of significant destruction or synthesis of tocopherol in the intestinal tract.

Fractionation of fecal tocopherol. No tocopheryl esters could be demonstrated in the feces, either during the control period, or during periods of high tocopheryl acetate intake. Thus, 71 to 80

TABLE IX

Recovery of tocopherol in feces following saponification

\begin{tabular}{|c|c|c|c|c|c|}
\hline Subject & Period & $\begin{array}{l}\text { dl-alpha } \\
\text { tocopheryl } \\
\text { acetate } \\
\text { ingested }\end{array}$ & $\begin{array}{l}\text { Tocopherol } \\
\text { recovered } \\
\text { on distil- } \\
\text { lation }\end{array}$ & $\begin{array}{r}\text { Tocophe } \\
\text { following }\end{array}$ & $\begin{array}{l}\text { rol recovered } \\
\text { saponification }\end{array}$ \\
\hline Cirrhotic & I & mg. & $\begin{array}{l}\text { mg. } \\
12.42\end{array}$ & $\begin{array}{l}\text { me. } \\
9.76\end{array}$ & $\begin{array}{c}\text { per cent of } \\
\text { amount found } \\
\text { on distillation } \\
78.5\end{array}$ \\
\hline Normal & $\begin{array}{l}\text { I } \\
\text { II } \\
\text { III } \\
\text { IV } \\
\text { V } \\
\text { VI }\end{array}$ & $\begin{array}{r}0 \\
1,700 \\
1,800 \\
500 \\
0 \\
0\end{array}$ & $\begin{array}{r}69.81 \\
1,138.80 \\
1,115.92 \\
429.51 \\
72.99 \\
65.79\end{array}$ & $\begin{array}{r}50.57 \\
916.62 \\
807.53 \\
321.44 \\
53.40 \\
47.03\end{array}$ & $\begin{array}{l}72.4 \\
80.5 \\
72.4 \\
74.8 \\
73.2 \\
71.5\end{array}$ \\
\hline Normal & $\begin{array}{l}\text { I } \\
\text { II } \\
\text { III } \\
\text { IV } \\
\text { V } \\
\text { IV }\end{array}$ & $\begin{array}{r}0 \\
1,700 \\
1,800 \\
500 \\
0 \\
0\end{array}$ & $\begin{array}{r}48.62 \\
954.76 \\
1,150.12 \\
354.45 \\
58.19 \\
55.30\end{array}$ & $\begin{array}{r}36.02 \\
726.64 \\
877.31 \\
259.90 \\
41.55 \\
40.43\end{array}$ & $\begin{array}{l}74.1 \\
76.1 \\
76.3 \\
73.3 \\
71.4 \\
73.1\end{array}$ \\
\hline
\end{tabular}

Recovery of dl-alpha-tocopheryl acetate added to $100 \mathrm{gm}$. aliquots of feces

$\begin{array}{ccc}\begin{array}{c}\text { Amount added } \\ \text { ms. }\end{array} & \begin{array}{c}\text { Recovered following saponification } \\ \text { mg. }\end{array} & \text { per cent } \\ 10.00 & 7.21 & 72.1 \\ 25.00 & 18.47 & 74.0 \\ 50.00 & 37.00 & 74.0 \\ 100.00 & 76.34 & 76.3 \\ 500.00 & 386.40 & 77.2 \\ 1,000.00 & 787.40 & 78.7\end{array}$

TABLE $X$

Stability of tocopheryl acetate and esterase activity in incubated jejunal contents* aspirated from a subject with intestinal obstruction; dl-alpha-tocopheryl acetate added in a concentration of 2,000 mg. per $100 \mathrm{ml}$. (equivalent to $1,828 \mathrm{mg}$. per cent of free tocopherol)

\begin{tabular}{|c|c|c|c|c|}
\hline $\begin{array}{l}\text { Incuba- } \\
\text { tion } \\
\text { at } \\
37^{\circ} \mathrm{C}\end{array}$ & \multicolumn{2}{|c|}{$\begin{array}{l}\text { Stability of tocopheryl } \\
\text { acetate }\end{array}$} & \multicolumn{2}{|c|}{ Esterase activity } \\
\hline hours & $\begin{array}{l}\text { mg. free tocopherol } \\
\text { recovered following } \\
\text { saponification }\end{array}$ & $\begin{array}{l}\text { per cent of } \\
\text { original } \\
\text { acetate } \\
\text { recooved }\end{array}$ & $\begin{array}{l}\text { mg. free tocopherol } \\
\text { recovered before } \\
\text { saponification }\end{array}$ & $\begin{array}{l}\text { per cent of } \\
\text { original } \\
\text { acetate } \\
\text { hydrolyzed }\end{array}$ \\
\hline $\begin{array}{r}1 \\
2 \\
4 \\
8 \\
24 \\
48\end{array}$ & \begin{tabular}{|l|}
1,689 \\
1,683 \\
1,689 \\
1,677 \\
1,665 \\
1,653
\end{tabular} & $\begin{array}{l}92.4 \\
92.1 \\
92.4 \\
91.7 \\
91.1 \\
90.4\end{array}$ & \begin{tabular}{r|}
50 \\
165 \\
368 \\
646 \\
1,161 \\
1,347
\end{tabular} & $\begin{array}{r}2.7 \\
9.0 \\
20.1 \\
35.3 \\
63.5 \\
73.7\end{array}$ \\
\hline
\end{tabular}

*Original content of free tocopherol $0.24 \mathrm{mg}$. per cent.

per cent of the original tocopherol found on distillation was recovered following saponification of the feces, values which compared favorably with recoveries following saponification of pure dlalpha-tocopherol added to feces (Table IX).

Destruction of tocopherol in feces and intestinal contents. M. K., a female cirrhotic with a plasma tocopherol level of $0.80 \mathrm{mg}$. per cent, excreted $443.23 \mathrm{mg}$. of tocopherol in a three-day period during which she ingested $1,800 \mathrm{mg}$. of dl-alphatocopheryl acetate. Following incubation of the feces in a fine watery suspension at $37^{\circ} \mathrm{C}$. for 24 hours, there was no loss of tocopherol, $454.62 \mathrm{mg}$. being recovered.

The contents of the lower jejunum were aspirated in N. R., a middle-aged negress with complete intestinal obstruction, who had no food by mouth and who had been maintained on tocopherol-free parenteral fluids for a period of two weeks. The fluid had a $\mathrm{pH}$ of 6.5 and contained $0.24 \mathrm{mg}$. per cent of tocopherol. Following incubation at $37^{\circ} \mathrm{C}$. for 24 hours the concentration fell insignificantly to $0.22 \mathrm{mg}$. per cent. When dl-alpha-tocopheryl acetate was added at a concentration of $2.0 \mathrm{gm}$. per cent, the ester was promptly broken down to free tocopherol, but there was no significant loss of tocopherol even after 48 hours of incubation (Table $\mathrm{X}$ ).

The stability of tocopherol and its esters in incubated feces and jejunal fluid suggested that there was no significant destruction of tocopherol in the intestinal tract. Tocopherol synthesis was not excluded, but it could not have been a signifi- 
cant factor in any of the experimental periods when there was a five- to 20 -fold increase in fecal tocopherol following tocopherol administration. Moreover, there is no convincing evidence of tocopherol synthesis in experimental animals. Pindborg (13) reported that synthesis occurred in rats, but his animals were on a stock diet and the more likely possibility, that the tocopherol found in the feces represented an unabsorbed fraction of ingested tocopherol, was not considered.

Urinary excretion of tocopherol. The amount of tocopherol found in the urine was insignificant, even following the administration of large doses of tocopherol. Thus, L. B., a convalescent control, excreted a total of $0.40 \mathrm{mg}$. per day while on a normal diet, and $0.26 \mathrm{mg}$. following the ingestion of $1200 \mathrm{mg}$. of dl-alpha-tocopheryl acetate. Similarly, a cirrhotic, A. S., excreted $0.93 \mathrm{mg}$. per day during the control period and $0.56 \mathrm{mg}$. following the administration of a similar dose of tocopheryl acetate.

It was concluded that these negligible losses in the urine had no influence on the concentration of tocopherol in the plasma or feces, or on the results of the saturation and tolerance tests.

\section{COMMENT}

It is highly probable that low concentrations of tocopherol in the plasma are indicative of a similar decrease in the tissues. The sustained rise in the plasma level of cirrhotics following saturating doses of tocopherol (Table III), and the low concentrations of tocopherol found in cirrhotic liver tissue (2) are confirmatory evidence of such a relationship. The failure of saturating doses of tocopherol to produce a similar rise in plasma concentration in convalescents appears to be inconsistent, but the marked difference between the amounts of tocopherol absorbed by convalescents and cirrhotics during the saturation test (Tables VI and VII) probably accounts for this discrepancy.

The possible causes for tocopherol depletion in cirrhosis to be considered include impairment of absorption, increased utilization or destruction in the tissues and a decreased dietary intake of tocopherol.

The data on fecal excretion provide convincing evidence that there is no impairment of tocopherol absorption in cirrhosis, and the observation that continuous tocopherol administration produces as great an increase in plasma concentration in cirrhotics as in normal subjects (Table III) is confirmatory.

The validity of estimating tocopherol absorption from balance studies, based on measurements of intake and fecal excretion, rests on two assumptions, that there is no significant loss of tocopherol by excretion in the urine or by destruction in the intestinal tract, and that there is no significant enteric synthesis of tocopherol. The evidence presented satisfactorily excludes significant losses of tocopherol in the urine, but the results of the in vitro incubation experiments to rule out tocopherol destruction in the intestinal tract, while suggestive, are by no means conclusive and still need confirmation. The possibility that tocopherol is synthesized in the intestinal tract has not been ruled out, but appears unlikely. The only contradictory evidence (13) is not convincing and has not been confirmed. However, even if tocopherol synthesis should prove to be a significant factor, it is unlikely that it introduced any serious errors in the present balance experiments, since fecal tocopherol excretion was increased five- to 20 -fold over the basal level during periods of tocopherol administration (Table VI).

The curious observation that cirrhotics apparently absorb a larger fraction of administered tocopherol than normal subjects (Table VII) warrants careful consideration. Conceivably the amount of tocopherol absorbed might be determined by its concentration in the plasma and tissues, as in the case of iron. However, this appears unlikely in view of the fact that a similar increase in tocopherol absorption is not seen in convalescent controls with equally low plasma concentrations (Table VII). A more likely possibility to be considered is that a significant fraction of the tocopherol absorbed is re-excreted via the biliary tract, and that under conditions of impaired hepatic function there is retention of this fraction to a greater or lesser extent. Most of the observed facts are compatible with this interpretation. These include the smaller fecal excretion of tocopherol in cirrhotics than in normal or convalescent controls, the greater effect of saturating doses of tocopherol on the plasma level in cirrhotics than in convalescents with similarly depleted tissue stores, the unusually large amount 
of tocopherol excreted immediately following saturation in all groups (Period V, Tables VI and VII) and the persistence of this increased excretion for a longer period in cirrhotics than in normals or convalescents (Period VI, Tables VI and VII). Only the tendency toward flat plasma curves following a single test dose of tocopherol in cirrhosis appears to be inconsistent with the hypothesis of normal intestinal absorption and impaired biliary excretion of tocopherol. However, it is possible that this trend merely indicates a slower rate of absorption in cirrhosis, as suggested by the fact that the areas under the tolerance curves are normal, or a more rapid diversion of tocopherol from the plasma to relatively unsaturated tissues, as suggested by the more normal curves following saturation.

A preliminary investigation of the biliary excretion of tocopherol was carried out in four subjects with $\mathrm{T}$-tube drainage of the common bile duct, to obtain more direct evidence of the reexcretion of absorbed tocopherol. It was found that the concentration of tocopherol in bile was of the same order of magnitude as in plasma, that the two concentrations varied independently, and that there was no increase in the bile concentration following the administration of large doses of tocopherol. These findings are consistent with the concept that a fraction of the tocopherol absorbed in the intestinal tract is re-excreted in the bile. However, it is not certain that the failure of the biliary concentration to rise following tocopherol administration was due to abnormal retention related to liver damage, since it was not possible to repeat this experiment in a normal subject.

The possibility that the low plasma tocopherol levels in cirrhosis are related to an increased rate of utilization or destruction of tocopherol appears unlikely in view of the sustained rise in the plasma level which follows saturating doses of tocopherol, and the lack of any correlation between the plasma level and the degree of hepatic dysfunction. On the other hand, these observations, and others, are compatible with the hypothesis that the low plasma tocopherol levels in cirrhosis are indicative of depleted tissue stores related to a decreased dietary intake of tocopherol. Certainly signs of other nutritional deficiencies are frequently observed in cirrhosis, and it is reasonable to suppose that tocopherol deficiency may also occur as a result of the reduced intake of food which so often precedes and accompanies the development of this disease. Qualitative and quantitative differences in the diet could well account for the inconstancy of low plasma tocopherol levels in cirrhosis. Similarly the low levels found in some convalescents could be related to a reduced intake of food during a prolonged antecedent illness.

\section{SUM MARY}

1. The mean plasma tocopherol level was significantly lower in subjects with Laennec's cirrhosis than in normal healthy controls, but did not differ significantly from that of hospitalized, convalescent controls with no evidence of liver damage. Moreover, low values were not a constand finding in cirrhosis, and could not be correlated with the degree of hepatic dysfunction present, suggesting that they were probably related to extrahepatic factors.

2. Large doses of tocopherol, equivalent to the estimated normal total body content, administered over an eight-day period, raised the plasma tocopherol to equally high levels in cirrhotics, normals and convalescent controls. However, following cessation of tocopherol administration, only the cirrhotics exhibited a sustained increase in plasma tocopherol over the control level. These findings suggested that there was no impairment of tocopherol absorption in cirrhosis and that low plasma levels were indicative of relatively unsaturated tissue stores.

3. Suggestive evidence was presented to establish the validity of estimating tocopherol absorption from measurements of tocopherol intake and fecal excretion. On the basis of such balance studies it was found that cirrhotics absorbed a higher proportion of administered tocopherol than either normal or convalescent controls, confirming the previous suggestion that low plasma levels were not the result of impaired tocopherol absorption. Several possible explanations for the apparently greater absorption of tocopherol in cirrhotics than in the controls were considered, but the data presented did not warrant any definite conclusions.

4. Tocopherol tolerance tests, based on the plasma response to a single test dose of tocopherol, 
revealed that the cirrhotics tended to show curves that were lower than those of the controls, judging from the maximum increase in concentration over the basal level. However, when the curves were compared on the basis of the mean rise in concentration, estimated from the areas under the curves, the three groups did not differ significantly. Following saturation of the tissues with large doses of tocopherol no differences could be discerned between the three groups. Parallel fecal excretion studies indicated that flat tolerance curves could not be interpreted as evidence of impaired absorption. Other interpretations were considered, but it was not possible to reach any definite conclusions.

5. On the basis of the evidence presented it is suggested that low plasma tocopherol levels in cirrhosis are indicative of depleted tissue stores related to a reduced dietary intake of tocopherol.

\section{REFERENCES}

1. Klatskin, G., and Krehl, W. A., The significance of the plasma tocopherol concentration and of tocopherol tolerance tests in liver disease. J. Clin. Invest., 1950, 29, 1528.

2. Popper, H., Dubin, A., Steigmann, F., and Hesser, F. P., Plasma tocopherol levels in various pathologic conditions. J. Lab. \& Clin. Med., 1949, 34, 648.
3. Harris, P. L., Quaife, M. L., and Swanson, W. J., Vitamin E content of foods. J. Nutrition, 1950, 40, 367.

4. Quaife, M. L., and Biehler, R., A simplified hydrogenation technique for the determination of blood plasma tocopherols. J. Biol. Chem., 1945, 159, 663.

5. Klatskin, G., and Molander, D. W., The chemical determination of tocopherols in feces, and the fecal excretion of tocopherol in man. To be published.

6. Quaife, M. L., and Harris, P. L., Chemical assay of foods for vitamin $\mathrm{E}$ content. Analyt. Chem., 1948, 20, 1221.

7. Wright, S. W., Filer, L. J., Jr., and Mason, K. E., Vitamin $\mathrm{E}$ blood levels in premature and full term infants. Pediatrics, 1951, 7, 386.

8. Quaife, M. L., and Dju, M. Y., Chemical estimation of vitamin $\mathrm{E}$ in tissue and the tocopherol content of some normal human tissues. J. Biol. Chem., 1949, 180, 263.

9. Engel, C., Vitamin $E$ in human nutrition. Ann. New York Acad. Sc., 1949, 52, 292.

10. Bratzler, J. W., Loosli, J. K., Krukovsky, V. N., and Maynard, L. A., Effect of the dietary level of tocopherols on their metabolism in swine. J. $\mathrm{Nu}$ trition, 1950, 42, 59.

11. Vinet, A., and Meunier, P., Sur le passage de tocophérol dans le sang et la possibilité d'un test direct d'avitaminose E. Compt. rend. Acad. d. sci., 1941, 213, 709.

12. Engel, C., Spaltung von Tocopherol-PhosphorsäureEster in vivo. Acta brev. Neerland., 1941, 11, 18.

13. Pindborg, J. J., Role of the intestinal flora in the development of vitamin $\mathrm{E}$ deficiency. Nature, 1949, $164,493$. 